\title{
Caries Dental en Personas con Retraso Mental y Síndrome de Down
}

\author{
María E. Dávila ${ }^{1}$, Maritza Gil², Damelis Daza ${ }^{3}$, Xiomara Bullones ${ }^{4}$ y Eunice Ugel ${ }^{5}$ \\ ${ }^{1}$ Odontólogo. Post doctorado en Salud Pública, Ph. D. Salud Pública, M. Sc. Salud Pública. Sección de Epidemiología \\ y Bioestadística. Escuela de Medicina de la Universidad Centroccidental Lisandro Alvarado, Barquisimeto, Venezuela. \\ E-mail: mangue98@hotmail.com; mdavila@ucla.edu.ve \\ ${ }^{2}$ Médico Cirujano. M. Sc. Salud Pública. Sección de Epidemiología y Bioestadística. Escuela de Medicina de la \\ Universidad Centroccidental Lisandro Alvarado, Barquisimeto, Venezuela. E-mail: mgil@ucla.edu.ve \\ ${ }^{3}$ Médico Cirujano. M. Sc. Salud Pública. Sección de Epidemiología y Bioestadística. Escuela de Medicina de la \\ Universidad Centroccidental Lisandro Alvarado, Barquisimeto, Venezuela. E-mail: djgarcia26@yahoo.com \\ ${ }^{4}$ Médico Cirujano. M. Sc. Salud. Sección de Epidemiología y Bioestadística. Escuela de Medicina de la Universidad \\ Centroccidental Lisandro Alvarado, Barquisimeto, Venezuela. E-mail: Xiomara_b@hotmail.com \\ ${ }^{5}$ Técnico Superior en Informática. Sección de Epidemiología y Bioestadística Escuela de Medicina de la Universidad \\ Centroccidental Lisandro Alvarado, Barquisimeto, Venezuela. E-mail: euniceu@hotmail.com \\ Recibido 4 Abril 2006/Enviado para Modificación 29 Julio 2006/Aceptado 3 Octubre 2006

\section{RESUMEN}

Objetivo Con el propósito de determinar la prevalencia de caries dental en personas con retraso mental (RM) y Síndrome de Down (SD) se realizó una investigación descriptiva transversal.

Métodos Se visitó la escuela especial ubicada en el Municipio Morán, Estado Lara, Venezuela, la cual cumplía con los criterios de inclusión, se realizó el diagnostico de salud bucal de 60 alumnos y entrevistó a los padres y/o representantes.

Resultados La edad promedio de los participantes fue de $14,5 \pm 8,17$ y 48,3 $\%$ pertenecen a la clase social obrero. En relación con la salud bucal $53 \%$ presentaba caries dental, de ellos $51,7 \%$ en los molares. Las personas con RM moderado presentaron el mayor porcentaje de caries dental $(75 \%)$. Aproximadamente $53 \%$ de las mujeres y $46,2 \%$ de los varones, $64,4 \%$ y 31,9 $\%$ de los participantes con RM y SD, tenían caries dental, respectivamente. Requerían tratamiento dental urgente $28,3 \%$ de los participantes, y $45 \%$ utilizaban los servicios odontológicos públicos.

Conclusiones Las personas con RM y SD presentan una precaria salud bucal por lo que se recomienda la creación de programas dirigidos a estas personas.

Palabras Clave: Retraso mental, Síndrome de Down, Caries dental, clase social (fuente: DeCS, BIREME)

\section{ABSTRACT \\ Dental caries amongst mentally retarded people and those suffering from Down's syndrome}

Objective A descriptive investigation was conducted for determining the prevalence of dental caries amongst mentally retarded (MR) people and those suffering from Down's syndrome (DS). 
Methods A special school located in Morán county in the State of Lara was visited. The inclusion criteria involved dental examination of 60 students and their parents/or the person responsible for them being interviewed.

Results Participants' mean age was $14,5 \pm 8,17 ; 48,3 \%$ were from a blue collar level. Regarding oral health, $53.0 \%$ had dental caries, 51,7 \% being in the molars. Moderately MR people had the highest proportion of dental caries (75\%). About $53 \%$ of the females and $46,2 \%$ of males, $64,4 \%$ and $31,9, \%$ of RM and DS participants had caries. $28,3 \%$ needed non-urgent dental treatment and $45 \%$ were using public dental health services.

Conclusions People suffering from MR and DS have precarious oral health; it is thus recommended that health programmes be created which are specifically directed towards these people.

Key Words: Mentally retarded, Down's syndrome, dental caries, social class (source: MeSH, NLM).

$\mathrm{E}$ 1 retraso mental (RM), incluyendo el Síndrome de Down (SD), puede afectar a personas todas las razas y clase social. De acuerdo con la Organización Mundial de la Salud (OMS) 3 \% de la población mundial tiene algún tipo de RM, siendo África y Asia los continentes con mayor prevalencia; por otra parte, Latinoamérica ocupa el quinto lugar en el mundo con 13800000 personas con RM (1).

Tradicionalmente, las personas con RM son aisladas del resto de sus congéneres, en lugar de ser estimulados a continuar con una vida plena y saludable. Sin embargo, en los últimos cuarenta años se ha visto un cambio en el comportamiento de la población hacia las personas con RM, además, poca atención se ha dedicado a resolver el estado de salud y necesidades de servicio en las personas con RM en los últimas cuatro décadas (2).

La meta en salud para el 2010 propuesta por la OMS, "Gente Sana para el 2010" (3), consiste en la reducción de las disparidades en salud de las poblaciones. Las disparidades en las condiciones de salud oral y acceso a los cuidados dentales en niños tienen una particular importancia epidemiológica, dado que existe una baja utilización de los servicios de salud en niños de cualquier edad siendo la caries dental la enfermedad de mayor prevalencia en este grupo poblacional.

Para los millones de personas en el mundo con RM y SD, el cuidado dental no es generalmente una prioridad, las complicaciones médicas de las que no escapa esta población, son tomadas en cuenta con mayor interés por 
los familiares y por las autoridades de salud. La atención bucodental es la única necesidad aún no cubierta en el tratamiento integral y rehabilitación de los pacientes con RM tanto en calidad como en cantidad. Los problemas dentales se encuentran entre las diez primeras causas que limitan la actividad de estas personas entre ellos la caries dental; pérdida dentaria y la enfermedad periodontal son las afecciones mas frecuentes en esta población $(4,5)$.

El desconocimiento de la situación en el Estado Lara, ha motivado la realización de una investigación que permita conocer el estado de salud oral de las personas con RM y SD en los municipios donde existan escuela especiales, siendo uno de ellos el Municipio Morán, con la finalidad de proponer a las autoridades de salud del estado, programas de atención en salud oral en los ambulatorios y hospitales regionales, dirigidos a esta población especial.

\section{MATERIALES Y MÉTODOS}

Con el propósito de determinar la prevalencia de caries dental de las personas con RM se realizó una investigación descriptiva transversal. Se utilizó una muestra probabilística de conglomerados, con un muestreo bietápico. En la primera etapa, se conformaron conglomerados constituidos por las escuelas especiales ubicadas en siete Municipios, en las que se atienden cuatrocientas personas con diversas patologías (Retraso Mental Leve-Moderado incluyendo Síndrome de Down), resultando 88 conglomerados. Para la selección de los conglomerados a incluir, se utilizó el muestreo aleatorio simple con un nivel de confianza de $95 \%$, un error de estimación de muestreo de 0,05 y una probabilidad de encontrar problemas de salud oral en las personas con retraso mental de 0,40. La muestra quedó conformada por 15 escuelas especiales. En la segunda etapa, se seleccionó la muestra de personas con RM que acudían a una escuela de educación especial seleccionada en el Municipio Morán y presentaran RM leve o moderado (incluyendo Síndrome de Down). La muestra quedó conformada por 60 personas. Se procedió a solicitar a los directores de las escuelas y a padres o representantes autorización para examinar a las personas con RM y SD. El examen oral fue practicado por un odontólogo previamente entrenado en el Seminario "Standarized Oral Health Screnning" (6) en el marco de las Olimpiadas Especiales de Invierno, Alaska, USA en Febrero 2001. Para ello se utilizó una unidad dental portátil, espejo y luz natural. La recolección de datos para el diagnóstico de salud oral se realizó

utilizando el instrumento elaborado por el Centro de Control de Enfermedades 
Infectocontagiosas de Atlanta (CDC), Departamento de Salud y Servicios Humanos de los Estados Unidos y utilizado en las Olimpiadas Especiales. Se entrevistó a los padres y/o representantes para obtener información sobre características demográficas.

Para el diagnóstico de los indicadores de salud oral se utilizaron los siguientes criterios: diente cariado sin tratar fue definido como: pieza dentaría con al menos un área erosionada de aproximadamente $0.5 \mathrm{~mm}$ de diámetro; diente obturado: pieza dentaría en la que cualquier trabajo dental se llevó a cabo exclusivamente como respuesta a la caries dental; diente perdido: como consecuencia de caries dental y urgencia de tratamiento basados en los hallazgos clínicos durante el examen oral.

El análisis de la información se realizó con el paquete estadístico SPSS versión 11.5, utilizando promedios, desviación estándar, porcentajes y la prueba de Chi cuadrado para determinar la asociación entre las variables en estudio.

\section{RESULTADOS}

Entre los hallazgos de esta investigación se evidenció que del total de personas con RM y SD participantes 50 \% eran del sexo masculino y el otro $50 \%$ del sexo femenino, con edad promedio de 14 años de edad. Con respecto al tipo de RM y SD 63,3 \% de los participantes tienen RM leve y 15,0 \% SD leve y $12,0 \%$ pertenecían al grupo de SD moderado (Tabla 1 ). Cuarenta y ocho por ciento (48,3 \%) de los participantes pertenecen a la clase social "obrero" y $21,7 \%$ a la clase social "medio, bajo y marginal”, respectivamente. En relación a la presencia de caries dental según tipo de RM fue mayor en el RM moderado, seguido de RM leve, (Figura 1). El mayor porcentaje de personas sin caries dental se encuentra entre el SD leve (77,8\%) seguido del SD moderado $(57,4$ $\%)$. Al analizar la presencia de caries dental según sexo se evidenció que 46,9 $\%$ del sexo femenino y 53,1 \% del sexo masculino tenía caries dental. Se observó que un porcentaje considerable de personas había perdido por caries dental el primer molar y aproximadamente $2 \%$ había perdido los dientes anteriores (Tabla 2). Al investigar el tipo de consulta odontológica al que acuden las personas con RM y SD se determinó que $45 \%$ asisten a la consulta pública, $33 \%$ a la consulta privada y $22 \%$ a ningún tipo de consulta. Al relacionar la presencia de caries dental y RM/SD, se encontró diferencia estadísticamente significativa ( $\mathrm{chi}^{2}=4,27$, valor de $\mathrm{p}<0,038$ ); lo opuesto se observó entre caries dental y sexo $\left(\mathrm{chi}^{2}=0,268\right.$ valor de $\left.\mathrm{p}=0,605\right)$. 
Tabla 1. Distribución de las personas según tipo de RM y SD

\begin{tabular}{lcc}
\hline \multicolumn{1}{c}{ Tipo de retraso } & No. & Porcentaje \\
\hline RM leve & 38 & 63,3 \\
RM moderado & 4 & 6,7 \\
RM severo & 2 & 3,3 \\
Síndrome de Down Leve & 9 & 15,0 \\
Sindrome de Down Moderado & 7 & 11,7 \\
\hline \multicolumn{1}{c}{ Total } & 60 & 100 \\
\hline
\end{tabular}

Fiqura 1. Prevalencia de caries dental seqún tipo de RM y SD.

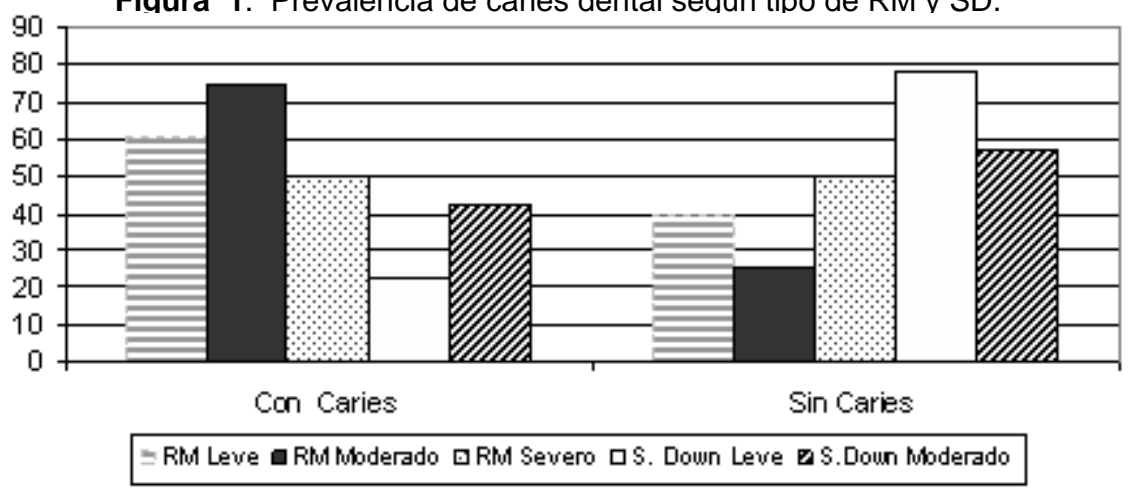

Tabla 2. Distribución de las personas con RM según diente extraído por caries dental

\begin{tabular}{ccccccc}
\hline $\begin{array}{c}\text { Diente } \\
\text { extraído }\end{array}$ & \multicolumn{2}{c}{ Anteriores } & \multicolumn{2}{c}{ 1er Molar } & \multicolumn{2}{c}{ Ninguno } \\
\hline & $\mathrm{N}^{0}$ & Porcentaje & $\mathrm{N}^{\circ}$ & Porcentaje & $\mathrm{N}^{\circ}$ & Porcentaje \\
\hline $\mathrm{Si}$ & 1 & 1,7 & 9 & 15,0 & 5 & 8,3 \\
$\mathrm{No}$ & 59 & 98,3 & 51 & 85,0 & 55 & 91,7 \\
\hline Total & 60 & 100,0 & 60 & 100,0 & 60 & 100,0 \\
\hline
\end{tabular}

\section{DISCUSIÓN}

La necesidad de tratamiento, la presencia de caries dental y la falta de higiene oral en personas con RM han sido estudiadas en diversos países. Gizani (7), en su estudio sobre la salud oral en personas discapacitadas, reporta que $21 \%$ estaban libres de caries dental. Con relación a la dentición permanente, 64,1 \% presentó caries en el primer molar permanente y 31,8 \% una pobre higiene oral, de ellos 22,1 \% correspondía a las personas con RM moderado. En la presente investigación, los resultados muestran que aquellos personas con RM moderado y leve presentaban el más alto porcentaje de caries dental (75 \% y 60,5 \%, respectivamente). Valores ligeramente menores a los anteriores fueron encontrados en cuatro municipios estudiados por Dávila (8). Por el contrario, 
la prevalencia de la caries dental es menor a los de Martín (9) en donde $79 \%$ de las personas con RM presentaban caries dental.

En cuanto al número de dientes anteriores y molares perdidos o extraídos, se observó que 1,7 \% y 15 \% de las personas con RM habían perdido dientes anteriores y molares, respectivamente. En el estudio realizado en Suiza (10), las personas con RM perdieron 3,72 piezas dentarias en 10 años. Estos resultados podrían indicar la capacidad de los diferentes estados para educar a los individuos con RM, así como la responsabilidad no sólo de los familiares e instituciones, sino también la responsabilidad social en la profesionalización de odontólogos y personal auxiliar en el cuidado y tratamiento de estas personas especiales.

Los hallazgos revelan que 33,3 \% acudían los servicios odontológicos privados, $45 \%$ a los servicios públicos y aproximadamente $22 \%$ a ningún tipo de servicio. Estas cifras son superiores a las reportadas por $\mathrm{Hu}$ (11) y menores a los encontrados en Inglaterra (12), donde se demostró que las visitas a los servicios odontológicas correspondían al 77 \% del total de visitas a los servicios de salud.

Al analizar el municipio según los indicadores de caries dental, se observó un alto porcentaje de las personas con RM tenían caries dental. En cuanto al tratamiento odontológico requerido, un considerable porcentaje de las personas requerían tratamiento dental urgente. Si bien no se encontró diferencia estadísticamente significativa en cuanto a clase social y caries dental, es importante destacar que la clase social que prevaleció en el municipio fue la obrera; este aspecto, aunado a la condición física de las personas con RM y la no utilización de los servicios, nos permitiría especular sobre la alta prevalencia de caries encontrada en el estudio

\section{REFERENCIAS}

1. ¿Que es el retardo mental? [Internet]. Disponible en: http://www.ongactitud.org/ preguntas.html. Consultado: Marzo 15 de 2005.

2. Horwitz S, Kerker B, Zigler E. Health Service use for individuals with mental retardation and summary recommendation. Prevalence of Mental Retardation. In: The Health Status and Needs of Individuals with Mental Retardation. Chapter 6. Washington, DC: Special Olympics, Inc.; Marzo 2001.

3. Healthy People 2010. US Dept of Health and Human Services; Washington, DC: Conference edition; January 2000. 
4. Horwitz S, Kerker B, Zigler E. Dental health among individuals with mental retardation. In: The Health Status and Needs of Individuals with Mental Retardation. Chapter 5. Washington, DC Special Olympics, Inc; Marzo 2001.

5. Horwitz S, Kerker B, Zigler E. Prevalence of Mental Retardation. In: The Health Status and Needs of Individuals with Mental Retardation. Special Olympics, Inc. Washington, DC; Marzo 2001. pp 12-18.

6. White J, Beltrán E. Training Manual for Standarized Oral Screening. Division of Oral Health. National Center for Chronic Disease Prevention and Health promotion. Center for Disease Control and Prevention. US Department of Health and Human Services. Revised Edition; Spring 2000.

7. Gizani S, Declerk D, Vinckier F, Martens L, Marks L, Goffin G. Oral health condition of 12-year-old handicapped children in Flanders (Belgium). Community Dent Oral Epidemiol.1997; 25(5):352-7.

8. Dávila ME, Gil M, Daza D, Bullones X, Ugel E. Salud Oral de las personas con retraso mental de cuatro municipios del Estado Lara, 2003. Acta Odontol. Venezolana 2005, 43 (3):275-281.

9. Martin J, Andrés M, Borges M, Rodríguez O. Estado de salud bucal de los niños retrasados mentales de la escuela especial Félix Valera. Rev Cubana Estomatol 1998, 26(4):255-267.

10. Gabre P, Gahnberg L. Dental health status of mentally retarded adults with various living arrangements. Spec Care Dentist. 1994 Sep-Oct; 14(5):203-7.

11. Hu SW, Kao T, Liao PH. An Epidemiological Study of Dental Health, Treatment Needs, and Associated Factors in Children with Mental Retardation. [Internet]. Disponible en: http://iadr.confex.com/iadr/2003Goteborg/techprogram/ abstract_32397.htm. Consultado: 21 de Noviembre 2003.

12. Cumella S, Ransford $\mathrm{N}$. Needs for oral care among people with intellectual disability. Journal of Intellectual Disability Research. 2000; 44(3\&4):1-2. 